\title{
Growth Hormone Deficiency in a Child with Neurofibromatosis-Noonan Syndrome
}

\author{
Doğuş Vurallı1, Nazlı Gönç1, Dominique Vidaud2,3, Alev Özön1, Ayfer Alikaşifoğlu1, Nurgün Kandemir1 \\ ${ }^{1}$ Hacettepe University Faculty of Medicine, Department of Pediatric Endocrinology, Ankara, Turkey \\ 2EA7331, Université Paris Descartes, Sorbonne Paris Cité, Faculté de Pharmacie de Paris, Paris, France \\ ${ }^{3}$ Service de Biochimie et de Génétique Moléculaire, Hôpital Cochin, Assistance Publique-Hôpitaux de Paris, Paris, France
}

\section{WHAT IS ALREADY KNOWN ON THIS TOPIC?}

Neurofibromatosis-Noonan syndrome (NFNS) is a distinct entity which has variable features of both NFI (neurofibromatosis I) and Noonan syndrome (NS). Mutations in the NFI gene were identified in majority of NFNS cases. Growth hormone deficiency (GHD) has been relatively frequently identified in NFI and NS, there is limited experience with GHD in NFNS cases.

\section{WHAT THIS STUDY ADDS?}

Short stature is a feature of NFNS; however, in some cases it can be caused by GHD and patients with NFNS who are not growing sufficiently should be evaluated for GHD. The case presented herein had clinical features of NFNS with a mutation in the NFI gene. It is the first NFNS case reported in the literature with $\mathrm{GHD}$, receiving growth hormone $(\mathrm{GH})$ treatment, and reaching a successful final height under $\mathrm{GH}$ treatment.

\section{ABSTRACT}

Neurofibromatosis-Noonan syndrome (NFNS) is a distinct entity which shows the features of both NF1 (neurofibromatosis 1) and Noonan syndrome (NS). While growth hormone deficiency (GHD) has been relatively frequently identified in NF1 and NS patients, there is limited experience in NFNS cases. The literature includes only one case report of a NFNS patient having GHD and that report primarily focuses on the dermatological lesions that accompany the syndrome and not on growth hormone $(\mathrm{GH})$ treatment. Here, we present a 13-year-old girl who had clinical features of NFNS with a mutation in the NF1 gene. The case is the first NFNS patient reported in the literature who was diagnosed to have GHD and who received GH treatment until reaching final height. The findings in this patient show that short stature is a feature of NFNS and can be caused by GHD. Patients with NFNS who show poor growth should be evaluated for GHD.

Keywords: Growth hormone deficiency, growth hormone, neurofibromatosisNoonan syndrome, NF1 gene, neurofibromatosis type 1, Noonan syndrome

Conflict of interest: None declared

Received: 19.04 .2015

Accepted: 27.09 .2015

\section{Introduction}

Neurofibromatosis-Noonan syndrome (NFNS, OMIM 601321) was first defined as a distinct entity in 1985 by Allanson et al (1) who reported four unrelated patients with neurofibromatosis (NF) who also had manifestations of Noonan syndrome (NS). These cases had presented with clinical findings such as short stature, ptosis, midfacial hypoplasia, webbed neck, learning disabilities, and muscle weakness (1). Opitz and Weaver (2) also reported a similar syndrome, defined as a separate clinical entity which they named NFNS. This entity bore the features of both NF type 1 (NF1) and NS. These early reports were followed by others $(3,4,5,6,7,8,9,10,11)$. When the genetic studies performed on

Address for Correspondence

Doğuş Vurallı MD, Hacettepe University Faculty of Medicine, Department of Pediatric Endocrinology, Ankara, Turkey Phone: +90 3123051124 E-mail: dvuralli@hotmail.com

OJournal of Clinical Research in Pediatric Endocrinology, Published by Galenos Publishing. 
NFNS are reviewed, it is noted that a mutation was identified in the NF1 gene in the majority of these studies. The co-occurrence of NF1 and PTPN11 mutations has been shown in very few studies and has been attributed to a de novo mutation either in NF1 or PTPN11 gene $(12,13)$. Today, the opinion that NFNS originates from different mutations at distinct genes affecting a common intracellular signal transduction pathway called RAS-MAPK (mitogen-activated protein kinase) pathway is more widely accepted. This pathway plays roles in cell proliferation, differentiation, and apoptosis. The number of affected genes in the RAS-MAPK pathway and the diversity of the mutations in these genes result in various different phenotypic characteristics and different syndromes. Since these syndromes are associated with the effects on the same pathway, they are called "RASopathies" or RAS-MAPK syndromes and NFNS is an important RASopathy.

Growth hormone deficiency (GHD) has been relatively frequently identified in NF1 and NS patients. Those receiving growth hormone $(\mathrm{GH})$ treatment have been published as case reports and the growth pattern, GH responses, near-adult, and adult heights of these cases have been reported $(14,15,16,17)$. However, the literature includes only one study that shows GHD in NFNS cases and that report primarily focuses on the dermatological lesions that accompany NFNS (18). GH treatment in NFNS is still a matter of debate. To our knowledge, the case presented herein is the first reported NFNS patient with GHD who received $\mathrm{GH}$ treatment and was followed until she reached final height under $\mathrm{GH}$ treatment.

\section{Case Report}

A 13-year-old girl presented with short stature. Physical examination showed dysmorphic facial features, a short and

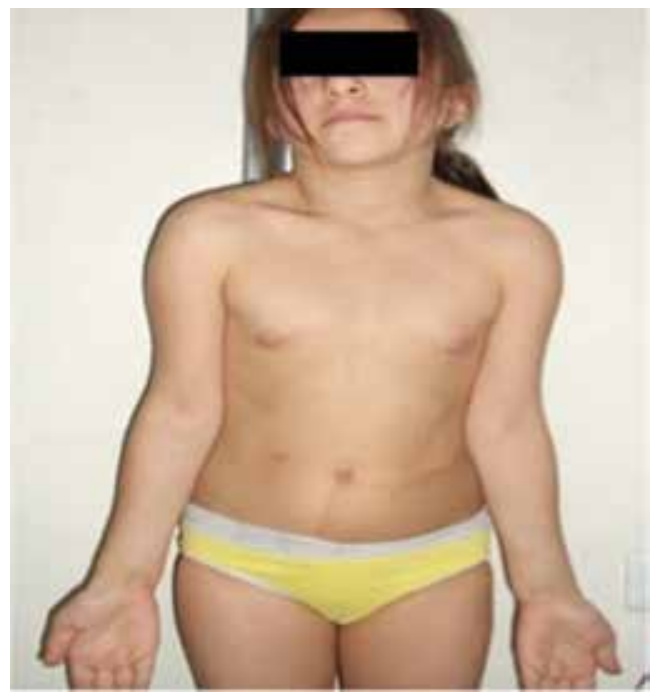

Figure 1. Physical findings of the patient suggestive of both neurofibromatosis 1 and Noonan syndrome webbed neck, low posterior hairline, cubitus valgus, brachyand clinodactyly, and widely spaced nipples suggesting NS and multiple café-au-lait spots (>15 mm, 8 spots), axillary freckling, and relative macrocephaly suggesting NF1 syndrome. Dysmorphic facial features included midfacial hypoplasia, prominent nasolabial folds, low-set and posteriorly rotated ears, hypertelorism, downslanted palpebral fissures, and low nasal root (Figure 1). The patient did not have any neurofibroma. Cardiovascular examination revealed no cardiac murmur and echocardiography was normal. The ocular examination did not reveal Lisch nodules. There was no sign of developmental delay, and the nervous system examination was completely normal. The patient's pubertal stage was evaluated as Tanner stage 2. Her arm span was $124.8 \mathrm{~cm}$ and upper/lower ratio was 0.9 suggesting no skeletal deformity. Karyotype analysis was $46, X X$. The auxological parameters of the case at diagnosis are given in Table 1.

Complete blood count, routine biochemistry, and urine analysis were within the normal limits. The celiac antibodies were negative and thyroid function tests were normal. Both serum insulin-like growth factor-1 (IGF-1) and IGF binding protein 3 (IGF-BP3) levels were below -3 standard deviation score (SDS). Peak GH response to L-dopa and clonidine stimulation tests were $3.9 \mathrm{ng} / \mathrm{mL}$ and $4.2 \mathrm{ng} /$ $\mathrm{mL}$, respectively. Other pituitary hormone levels were all within normal ranges. The serum pituitary hormone levels at diagnosis are given in Table 2 .

The size of the pituitary gland was measured as 3.5 $\mathrm{mm}$ in the pituitary magnetic resonance imaging (MRI) and this was considered to be consistent with anterior pituitary hypoplasia according to the age group of the patient. NFNS syndrome was suspected, and cranial MRI was performed to evaluate the neurological involvement. Cranial $\mathrm{MRI}$ showed a hyperintense mildly swollen appearance in T2 at the cerebral peduncles and globus pallidus that may be attributed to NF. T1-weighed images also showed hyperintense lesions associated with T1 limitation; after administration of intravenous contrast substance, these lesions did not show any uptake of the contrast (Figure 2).

Since the patient fulfilled the criteria of GHD, GH therapy was initiated with a dosage of $0.3 \mathrm{mg} / \mathrm{kg} /$ week. The height

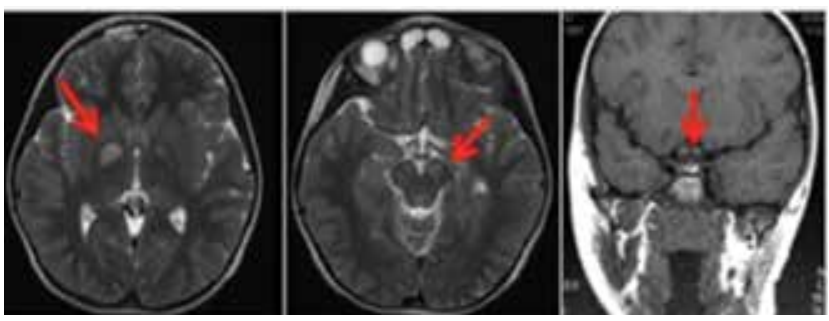

Figure 2. Cranial magnetic resonance imaging of the patient showing the lesions of neurofibromatosis in the cerebral peduncles and globus pallidus and adenopituitary hypoplasia in the pituitary 
velocity during the first year of $\mathrm{GH}$ treatment was $9.8 \mathrm{~cm} /$ year and was $7.2 \mathrm{~cm}$ and $4.5 \mathrm{~cm}$ on the second and third years of GH therapy, respectively. The patient's height was $147.3 \mathrm{~cm}$ (height SDS: -2.3 ) at the end of the third year of $\mathrm{GH}$ therapy. The patient was 16.5 years old when the GH therapy was discontinued; she had had three regular

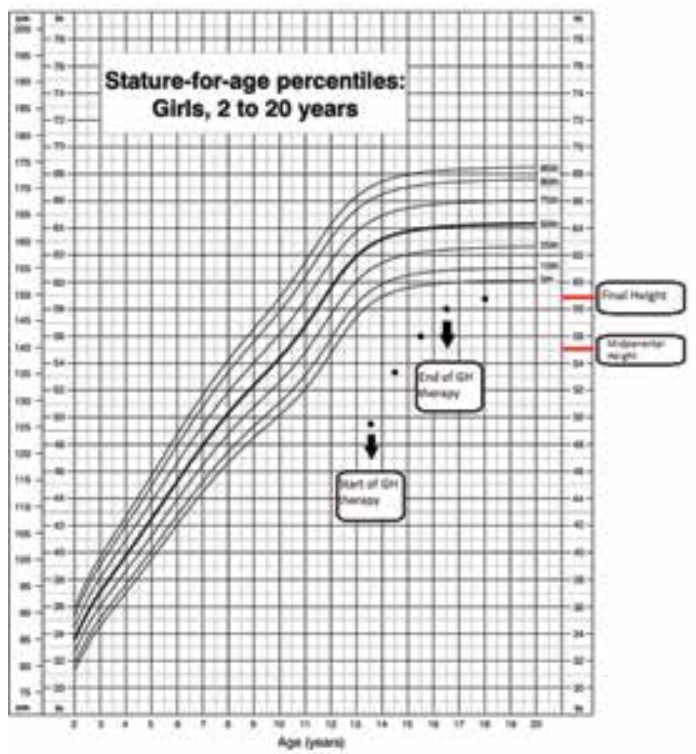

Figure 3. Growth chart of the patient under growth hormone therapy. Source: http:/www.cdc.gov/growth charts (Centers for Disease Control and Prevention (CDC) 2000)

\begin{tabular}{|l|l|}
\hline \multicolumn{2}{|l|}{ Table 1. Auxological data of the case at the time of diagnosis } \\
\hline Auxological parameters & \\
\hline Age at diagnosis (years) & 13.5 \\
\hline Bone age (years) & 11 \\
\hline Height (cm) & 125.8 \\
\hline Height SDS & -4.8 \\
\hline Weight (kg) & 26.3 \\
\hline BMI (kg/m2) & 16.6 \\
\hline BMI-SDS & -0.94 \\
\hline Height velocity at the beginning of GH therapy (cm/yr) & 1.3 \\
\hline Height velocity SDS at the beginning of GH therapy (cm/yr) & -2.8 \\
\hline Father's height (cm) & 150 \\
\hline Father's height SDS & -4.1 \\
\hline Mother's height (cm) & 145 \\
\hline Mother's height SDS & -2.9 \\
\hline Midparental height (cm) & 141 \\
\hline Midparental height SDS & -3.5 \\
\hline Predicted adult height (cm) & 137 \\
\hline Predicted adult height SDS & -4.2 \\
\hline SDS: standard deviation score, BMl: body mass index, GH: growth hormone \\
\hline
\end{tabular}

menstruation cycles and her bone age was 14.5 years. Her final height was $148.5 \mathrm{~cm}, 7.5 \mathrm{~cm}$ above the mid-parental height (Figure 3).

The patient's father also had features representing both NF1 and NS such as multiple café-au-lait spots, short stature, relative macrocephaly, and axillary freckling, suggesting NF syndrome, and findings such as prominent nasolabial folds, low-set ears, low nasal root, dysmorphic facial features, short neck, and cubitus valgus suggesting NS. It was learned that some members of the father's family also had multiple café-au-lait spots. Genetic analyses were performed for the patient and her father to investigate NFNS and the genetic analysis of both the patient and the father revealed a truncating mutation c.7846C $>\mathrm{T}$ (M82814), p.Arg2616X (AAA59924) in the NF1 gene. No mutation was found in PTPN11 gene.

\section{Discussion}

NFNS is an entity presenting with clinical characteristics of both NF1 and NS. The frequency of NFNS is thought to be higher than the current estimates, since these cases may be missed due to their being inadvertently diagnosed as classic NF1 or NS. In the study of Colley et al (11), the reassessment of 94 cases diagnosed with NF1 has demonstrated that 12 of these cases actually met the criteria for NS. This reassessment has shown that some

\begin{tabular}{|c|c|c|}
\hline & $\begin{array}{l}\text { Hormone levels } \\
\text { of the patient }\end{array}$ & $\begin{array}{l}\text { Reference } \\
\text { hormone levels }\end{array}$ \\
\hline $\mathrm{fT}_{4}(\mathrm{pmol} / \mathrm{L})$ & 12.8 & $7.86-14.41$ \\
\hline $\mathrm{fT}_{3}(\mathrm{pmol} / \mathrm{L})$ & 4.8 & $3.8-6.0$ \\
\hline $\mathrm{TSH}(\mu \mathrm{lU} / \mathrm{mL})$ & 2.31 & $0.34-5.6$ \\
\hline FSH (IU/L) & 1.6 & $>0.3$ (pubertal) \\
\hline LH (IU/L) & 0.5 & $>0.3$ (pubertal) \\
\hline Estradiol (pg/mL) & 22 & $>10$ (pubertal) \\
\hline ACTH $(\mathrm{pg} / \mathrm{mL})$ & 45.8 & $0-90$ \\
\hline Cortisol ( $\mu \mathrm{g} / \mathrm{dL})$ & 15.1 & $4.3-22.4$ \\
\hline PRL (ng/mL) & 12.1 & 3.3-18.7 \\
\hline IGF1 ( $\mu \mathrm{g} / \mathrm{L})$ & 150 & $203-831$ \\
\hline IGF-BP3 ( $\mu \mathrm{g} / \mathrm{L})$ & 2430 & $2710-6340$ \\
\hline $\begin{array}{l}\text { Peak GH level in L-dopa test } \\
\text { (ng/mL) }\end{array}$ & 3.9 & $\geq 10$ \\
\hline $\begin{array}{l}\text { Peak GH level in clonidine test } \\
\text { (ng/mL) }\end{array}$ & 4.2 & $\geq 10$ \\
\hline \multicolumn{3}{|c|}{$\begin{array}{l}\text { GH: growth hormone, } \mathrm{fT}_{4} \text { : free thyroxin } \mathrm{fT}_{3} \text { : free triiodothyronine, } \mathrm{TSH} \text { : thyroid } \\
\text { stimulating hormone, } \mathrm{FSH} \text { : follicle stimulating hormone, LH: luteinizing hormone } \\
\text { ACTH: adrenocorticotropic hormone, PRL: prolactin, IGF1: insulin-like growth factor-1 } \\
\text { IGF-BP3: insulin-like growth factor binding protein } 3\end{array}$} \\
\hline
\end{tabular}


Vurallı D et al.

Growth Hormone Deficiency in a Child with Neurofibromatosis-Noonan Syndrome

patients who have been clinically diagnosed with NF1 or NS can indeed be NFNS.

The genetic studies that have been undertaken to identify the gene causing NFNS have shown that the majority of these cases have a mutation in the NF1 gene. These studies have revealed that the mutations responsible for classic NF1 can also cause NFNS $(19,20,21)$. The mutation identified in the present case is indeed a mutation that is seen in classical NF1 cases. Additional studies are required to clarify which mutations cause classic NF1, which mutations cause NFNS, and which mutations have the potential to cause both.

Short stature is a common feature of NFNS as it is of NF1 and NS (22). The frequent causes of short stature in these syndromes are skeletal deformities and nutritional problems. Presence of suprasellar lesions is also a frequent cause, but GHD can develop in some of these patients in the absence of an underlying suprasellar lesion (15). While many studies have reported presence of GHD in NF1 and NS, to our knowledge, there is only one case report on NFNS receiving GH therapy (18). It is known that NS patients are of normal height and weight at birth and that growth deficiency develops later, with almost $80 \%$ of the cases eventually being of short stature (23). Several studies have shown that $\mathrm{GH}$ treatment increases the final height in NS cases $(24,25,26)$. Among these studies, the one with the highest number of cases is the National Cooperative Growth Study (27). This study involves a large cohort of 252 NS cases who have received GH therapy for 5.6 years on average. GHD can also be seen in NF1 cases in the absence of suprasellar lesions. Some researchers suggested that there could be a relationship between GHD and NF1 in the absence of an organic pituitary damage, and they agree that larger cohort studies are required to decide whether NF1 is a cause of GHD $(15,28)$. GHD-specific screening was recommended in NF1 cases with insufficient growth. An impairment in the cellular signal transduction was suggested as the reason of GHD in NF1 cases without suprasellar regions (29). Hegedus et al (30) showed that neurofibromin provides somatic growth by affecting the hypothalamic-pituitary axis. In their study, body weight and anterior pituitary gland size were found to decrease in mice with an inactivated NF1 gene. It was also shown that the decrease in anterior pituitary size reduces neurofibromin expression in the hypothalamus, thereby decreasing the production of $\mathrm{GH}$-releasing hormone, that of $\mathrm{GH}$ and IGF-1 as well.

There is only one case report in the literature about GHD and $\mathrm{GH}$ treatment in NFNS. As mentioned above, this one report focuses on the dermatological lesions in NFNS and gives no detailed information about $\mathrm{GH}$ treatment. Thus, the present manuscript is first to provide details of $\mathrm{GH}$ treatment in a NFNS case. The growth pattern of our patient showed that short stature had been a problem since early childhood, but that the problem had gradually increased within the last 2 years, during which her peers entered puberty and had pubertal growth. There was no underlying reason, such as severe skeletal deformities, suprasellar lesions, or nutritional deficiency to explain the short stature observed in the present case, thus initially, the short stature was considered to be related to the delay in puberty, as in NS cases. At admission, the height of our patient was $<-2$ SDS and her growth rate was very low. GHD was considered to be a possible reason for short stature, and $\mathrm{GH}$ stimulation tests were performed. The peak $\mathrm{GH}$ level was calculated as $<5 \mathrm{ng} / \mathrm{mL}$ in two $\mathrm{GH}$ stimulation tests suggesting that the patient had severe GHD. While the final height predicted based on bone age at the beginning of $\mathrm{GH}$ therapy was $137 \mathrm{~cm}$, the final height after $\mathrm{GH}$ therapy was $148.5 \mathrm{~cm}$. GH therapy resulted in an $11.5 \mathrm{~cm}(1.8 \mathrm{SDS})$ gain in the final height.

Recent studies indicate that NS and NF1 patients also benefit from $\mathrm{GH}$ treatment. In one study, the height gain based on CDC standards was $8.9 \mathrm{~cm}$ for boys and $10.0 \mathrm{~cm}$ for girls in NS cases receiving GH treatment and this gain was similar to that in Turner syndrome cases (27). In another study evaluating NF1 cases receiving GH treatment, the growth rate, which was $5 \mathrm{~cm} /$ year before $\mathrm{GH}$ therapy, increased to $9 \mathrm{~cm} /$ year at the first year of therapy, was $8.3 \mathrm{~cm} /$ year in the second year, and decreased to 6 $\mathrm{cm} /$ year between the third and the fifth years of treatment (15). Although the final height of our patient was $7.5 \mathrm{~cm}$ greater than the midparental height, it was still short due to the underlying familial short stature and the relatively short duration of $\mathrm{GH}$ therapy. The reason the father had a short stature was likely due to the fact that he also had NFNS, was not assessed with respect to GHD, and did not receive any treatment. When the patient was referred to our clinic, her puberty had already started. A better final height could possibly be achieved if $\mathrm{GH}$ treatment could have been started at a younger age. Studies have shown that the earlier that GH therapy is initiated in NF1 and NS cases, the better the final height that can be achieved $(14,15)$.

There is limited experience with GHD in NFNS cases, since it is a rare condition that is clinically difficult to identify. Our patient had the clinical features of NFNS and was found to have a mutation in the NF1 gene. She also had GHD and responded very well to $\mathrm{GH}$ treatment. It may be argued that short stature is a feature of NFNS, but it is evident that in some cases, short stature can be caused by GHD. For this reason, patients with NFNS who are not growing sufficiently should be evaluated for GHD. Those diagnosed to have GHD can benefit from GH treatment. However, it is obvious that more studies are needed on the use and benefits of GH therapy in NFNS cases, and also in NF1 and NS cases. 


\section{Ethics}

Informed Consent: It was taken.

Peer-review: External peer-reviewed.

\section{Authorship Contributions}

Concept: Doğuş Vurallı, Design: Doğuş Vurallı, Nazlı Gönç, Data Collection or Processing: Doğuş Vurallı, Dominique Vidaud, Analysis or Interpretation: Doğuş Vurallı, Nazlı Gönç, Dominique Vidaud, Alev Özön, Ayfer Alikaşifoğlu, Nurgün Kandemir, Literature Search: Doğuş Vurallı, Nazlı Gönç, Alev Özön, Ayfer Alikaşifoğlu, Nurgün Kandemir, Writing: Doğuş Vurallı, Nazlı Gönç.

Financial Disclosure: The authors declared that this study has received no financial support.

\section{References}

1. Allanson JE, Hall JG, Van Allen MI. Noonan phenotype associated with neurofibromatosis. Am J Med Genet 1985;21:457-462.

2. Opitz JM, Weaver DD. The neurofibromatosis-Noonan syndrome. Am J Med Genet 1985;21:477-490.

3. Kaplan $\mathrm{P}$, Rosenblatt B. A distinctive facial appearance in neurofibromatosis von Recklinghausen. Am J Med Genet 1985;21:463-470.

4. Mendez HM. The neurofibromatosis-Noonan syndrome. Am J Med Genet 1985;21:471-476.

5. Saul RA. Noonan syndrome in a patient with hyperplasia of the myenteric plexuses and neurofibromatosis. Am J Med Genet 1985;21:491-492

6. Meinecke P. Evidence that the "neurofibromatosis-Noonan syndrome" is a variant of von Recklinghausen neurofibromatosis. Am J Med Genet 1987;26:741-745.

7. Quattrin T, McPherson E, Putnam T. Vertical transmission of the neurofibromatosis/Noonan syndrome. Am J Med Genet 1987;26:645-649.

8. Shuper A, Mukamel M, Mimouni M, Steinherz R. Noonan's syndrome and neurofibromatosis. Arch Dis Child 1987;62:196198.

9. Abuelo DN, Meryash DL. Neurofibromatosis with fully expressed Noonan syndrome. Am J Med Genet 1988:29:937-941.

10. Stern HJ, Saal HM, Lee JS, Fain PR, Goldgar DE, Rosenbaum $\mathrm{KN}$, Barker DF Clinical variability of type 1 neurofibromatosis: is there a neurofibromatosis-Noonan syndrome? J Med Genet 1992;29:184-187.

11. Colley A, Donnai D, Evans DG. Neurofibromatosis/Noonan phenotype: a variable feature of type 1 neurofibromatosis. Clin Genet 1996;49:59-64.

12. Bertola DR, Pereira AC, Passetti F, de Oliveira PS, Messiaen L, Gelb BD, Kim CA, Krieger JE. Neurofibromatosis-Noonan syndrome: molecular evidence of the concurrence of both disorders in a patient. Am J Med Genet A 2005;136:242-245.

13. Thiel C, Wilken M, Zenker M, Sticht H, Fahsold R, GusekSchneider GC, Rauch A. Independent NF1 and PTPN11 mutations in a family with neurofibromatosis-Noonan syndrome. Am J Med Genet A 2009;149:1263-1267.

14. Noonan JA, Kappelgaard AM. The Efficacy and safety of growth hormone therapy in children with noonan syndrome: a review of the evidence. Horm Res Paediatr 2015:83:157-166. Epub 2014 Dec 10
15. Vassilopoulou-Sellin R, Klein MJ, Slopis JK. Growth hormone deficiency in children with neurofibromatosis type 1 without suprasellar lesions. Pediatr Neurol 2000;22:355-358.

16. Carmi D, Shohat M, Metzker A, Dickerman Z: Growth, puberty, and endocrine functions in patients with sporadic or familial neurofibromatosis type 1: a longitudinal study. Pediatrics 1999;103:1257-1262.

17. Kirk JM, Betts PR, Butler GE, Donaldson MD, Dunger DB, Johnston DI, Kelnar CJ, Price DA, Wilton P, Group tU: Short stature in Noonan syndrome: response to growth hormone therapy. Arch Dis Child 2001;84:440-443.

18. Reig I, Boixeda P, Fleta B, Morenoc C, Gamez L, Truchuelo M. Neurofibromatosis-Noonan syndrome: case report and clinicopathogenic review of the Neurofibromatosis-Noonan syndrome and RAS-MAPK pathway. Dermatology Online J 2011;17:4

19. Baralle D, Mattocks C, Kalidas K, Elmslie F, Whittaker J, Lees $\mathrm{M}$, Ragge N, Patton MA, Winter RM, ffrench-Constant C. Different mutations in the NF1 gene are associated with Neurofibromatosis-Noonan syndrome (NFNS). Am J Med Genet A 2003;119:1-8

20. De Luca A, Schirinzi A, Buccino A, Bottillo I, Sinibaldi L, Torrente I, Ciavarella A, Dottorini T, Porciello R,Giustini S, Calvieri S, Dallapiccola B. Novel and recurrent mutations in the NF1 gene in Italian patients with neurofibromatosis type 1. Hum Mutat 2004:23:629.

21. De Luca A1, Bottillo I, Sarkozy A, Carta C, Neri C, Bellacchio E, Schirinzi A, Conti E, Zampino G, Battaglia A,Majore S, Rinaldi MM, Carella M, Marino B, Pizzuti A, Digilio MC, Tartaglia M, Dallapiccola B. NF1 gene mutations represent the major molecular event underlying neurofibromatosis-Noonan syndrome. Am J Hum Genet 2005;77:1092-1101. Epub 2005 Oct 26

22. Nyström AM, Ekvall $S$, Allanson J, Edeby $C$, Elinder $M$, Holmström G, Bondeson ML, Annerén G. Noonan syndrome and neurofibromatosis type I in a family with a novel mutation in NF1. Clin Genet 2009;76:524-534. Epub 2009 Oct 21

23. Mendez HM, Opitz JM. Noonan syndrome: a review. Am J Med Genet 1985;21:493-506.

24. Osio D, Dahlgren J, Wikland KA, Westphal O. Improved fina height with long-term growth hormone treatment in Noonan syndrome. Acta Paediatr 2005;94:1232-1237.

25. Municchi G, Pasquino AM, Pucarelli I, Cianfarani S, Passeri F. Growth hormone treatment in Noonan syndrome: report of four cases who reached final height. Horm Res 1995;44:164-167.

26. Noordam C, Peer PG, Francois I, De Schepper J, van den Burgt I, Otten BJ. Long-term GH treatment improves adult height in children with Noonan syndrome with and without mutations in protein tyrosine phosphatase, non-receptor-type 11. Eur J Endocrinol 2008;159:203-208. Epub 2008 Jun 18

27. Romano AA, Dana K, Bakker B, Davis DA, Hunold JJ, Jacobs J, Lippe B. Growth response, near-adult height, and patterns of growth and puberty in patients with noonan syndrome treated with growth hormone. J Clin Endocrinol Metab 2009;94:23382344. Epub 2009 Apr 28

28. Cnossen MH, Stam EN, Cooiman LC, Simonsz HJ, Stroink $H$, Oranje AP, Halley DJ, de Goede-Bolder A, Niermeijer MF, de Muinck Keizer-Schrama SM. Endocrinologic disorders and optic pathway gliomas in children with neurofibromatosis type 1. Pediatrics 1997:100:667-670.

29. Marshall M. Interactions between Ras and Raf: key regulatory proteins in cellular transformation. Mol Reprod Dev 1995;42:493499.

30. Hegedus B, Yeh TH, Lee da Y, Emnett RJ, Li J, Gutmann $\mathrm{DH}$. Neurofibromin regulates somatic growth through the hypothalamic-pituitary axis. Hum Mol Genet 2008;17:2956-2966. Epub 2008 Jul 9 